\title{
Analysis of Anaphora Resolution System for English Language
}

\author{
Smita Singh, Priya Lakhmani, Dr. Pratistha Mathur, Dr. Sudha Morwal \\ Department of Computer Science \\ Banasthali University, Jaipur Campus \\ Jaipur, Rajasthan, India
}

\begin{abstract}
Anaphora resolution is complex problem in linguistics and has attracted the attention of many researchers. It is the problem of identifying referents in the discourse. Anaphora Resolution plays an important role in Natural language processing task. This paper completely emphasis on pronominal anaphora resolution for English Language in which pronouns refers to the intended noun in discourse. In this paper two computational models are proposed for anaphora resolution. Resolution of anaphora is based on various factors among which these models use Recency factor and Animistic Knowledge. Recency factor is implemented by using Lappin Leass approach in first model and using Centering approach in second model. Information about animacy is obtained by Gazetteer method. The identification of animistic elements is employed to improve the accuracy of the system. This paper demonstrates experiment conducted by both the models on different data sets from different domains. A comparative result of both the model is summarized and conclusion is drawn for the best suitable model.
\end{abstract}

\section{KEYWORDS}

Anaphora, Centering approach, Discourse, Gazetteer method, Lappin Leass approach

\section{INTRODUCTION}

Anaphora is a process of pointing back to previous item in the discourse. Discourse is a group of collocated and related sentences. The process of finding the referring expression to the correct antecedent, in the discourse, is called anaphora resolution or pronominal resolution. Consider the following:

"Anu bought a book. She likes reading."

This is an example of anaphora resolution. Here "She" refers to "Anu". However, the process of anaphora resolution can become increasingly complex when we encounter following sentence:

"Fruits were given to children because they were there."

In the above sentence "they" is either refer to "Fruits" or "children". This anaphor creates ambiguity \& resolves to either or both. Since this type of understanding is still poorly implemented in software, anaphora resolution is currently an area of active research. Introducing anaphora resolution to many applications like machine translation, opinion mining, automatic summarization, information extraction etc increases the accuracy of these applications to far extent.

The most common type of anaphora is the pronominal anaphora. It is the process of finding noun phrase which refers to pronoun and it occurs at the level of personal pronoun, possessive 
pronoun, demonstrative pronoun, reflexive pronoun and relative pronouns. This paper completely emphasis on pronominal anaphora resolution for English language

\section{RELATED WORK}

The work done for anaphora resolution in Indian and European Language is summarized below:

- Richard Evans and Constantin Orasan improved anaphora resolution by identifying animate entities in texts [4].

- Ruslan Mitkov, Richard Evans resolved anaphora resolution by using Gazetteer method in 2007[2].

- Tyne Liang and Dian-Song Wu used above approach in automatic pronominal anaphora resolution in English texts in 2002.

- Constantin Orasan and Richard Evans used NP Animacy Identification for Anaphora Resolution in 2007[2].

- Natalia N. Modjeska, Katja Markert and Malvina Nissim used web in Machine Learning for Other-Anaphora Resolution in 2003[3].

- Strube \& Hahn present a system for anaphora resolution for German based on extension of Centering theory in 1991[6].

- S. Lappin and H. Leass proposed their algorithm for pronoun resolution for English language in year 1994[7].

- Joshi, A. K. \& Kuhn. S, in 1979 and Joshi, A. K. \& Weinstein.S in 1981, gave centering theory for pronoun resolution [8].

- Dev Bahadur using Lappin Leass approach pronominal anaphora is resolved in Nepali Language[9].

- Thiago Thomes Coelho, Ariadne Maria Brito Rizzoni done work in Portugeese language using Lappin and Leass algorithm[7].

- Manuel Palomar, Lidia Moreno and Jesfis Peral resolved anaphora in Spanish Texts using Centering approach[10].

- S.Lappin and M.McCord developed a syntactic filter on pronominal anaphora for slot grammer using Lappin Leass principles in 1990[11].

- Sobha and Patnaik gave a rule based approach for the resolution of anaphora in Hindi and Malayalam as well[12].

- Dutta et al. presented modified Hobbs algorithm for Hindi[13].

- J.Balaji applied Centering principles in Tamil[14].

\section{APPROACH TO ANAPHORA RESOLUTION}

\subsection{Centering Theory}

Centering theory is a discourse based approach. Centering theory gives a framework to model what a sentence is speaking about. This can be used to find which entities are referred to by pronouns in a given sentence. This theory models the attentional salience of discourse entities, and relates it to referential continuity. Centering-based approaches are attractive from the computational point of view because the information they require can be obtained from structural properties of utterances alone, as opposed to costly semantic information. 


\subsection{Lappin Leass algo}

It is a hybrid approach. It use a model that calculates the discourse salience of a candidate based on different factors calculated dynamically and use this salience measure to rank potential candidates. The factors that the algorithm uses to calculate salience are given different weights according to how relevant the factor is. They do not use costly semantic or real world knowledge in evaluating antecedents.

\subsection{Gazetteer method}

It is used to provide external knowledge to the system. In this method lists are created. Elements present in the list are then classified based on certain operations. Therefore it is also called List Look Up method.

\section{RESOLVING SYSTEM}

\subsection{Salient Factors}

For resolving anaphora certain factors are to be considered. These factors are:

Recency: Recency factor describes that the referents mentioned in current sentence tends to have higher weights than those in previous sentence. For example consider the sentence,

"Riya dropped the plate. It shattered loudly."

In this sentence there are two nouns "Riya" and "plate". According to Recency factor the highest weight is assigned to the closest noun "plate" for the pronoun "It".

Animistic Knowledge: Animacy denotes the living entities which can be referred by some gendermarked pronouns (he, she, him, her, his, hers, himself, herself) in texts. Conventionally, animate entities include people and animals. Consider the following:

\section{"Neha bought pen for herself."}

In the above example pronoun "herself" is animistic pronoun (always refer to living things). So, "herself" refers to animistic noun "Neha".

In addition to Recency and Animistic factor, there are two more factors that affect the anaphora resolution. These are described as follows:

- Gender Agreement: Gender Agreement compares the gender of candidate co referents to the gender required by the pronoun being resolved. Any candidate that doesn't match the required gender of the pronoun is removed from further consideration.

"Anu bought a pen. She is a writer".

In the above example "She" refers to "Anu". In English pronouns such as "she", "he", "herself", "himself" are used to describe male and female.

- Number Agreement: Number Agreement extracts the part of speech of candidates. The part of speech label is checked for plurality. If the candidate is plural but the current 
pronoun being resolved doesn't indicate a plural co referent the candidate is removed from consideration. The same process occurs for singular candidates which are removed if the pronoun being resolved requires a plural co referent. This is an example of a constraint that relies on accurate part of speech tagging in the preprocessor.

"Niya and Riya are good friends. They both are painters."

In the above example, "They" refer to "Niya and Riya".

\subsection{Proposed Computational Model}

Two models are proposed based on two different approaches. In both the models, lists of animistic pronoun (pronoun refer to living things), non animistic pronoun (pronouns refer to non living things), middle animistic pronoun (pronouns refer to both living and non living things) are created. Lists of animistic noun (always represent living things) and non animistic noun (always represent non living things) are also created.

Computational Model 1: This model uses the concept of Centering approach for implementing Recency factor. Gazetteer method is used for providing animistic knowledge. When the system first encounters pronoun ,it check whether it is animistic, non animistic or middle animistic. If it is animistic then it will refer to animistic referent .If it is non animistic then it will refers to non animistic pronoun. If it is middle animistic then it will use the Centering approach(with Recency factor) for resolving pronoun. Centering theory is a discourse based method. In this method Recency is calculated by using Centering transitions. These transitions rules are used to resolve anaphors.

Computational Model 2: This model first check whether the pronoun falls under the category of animistic, non animistic or middle animistic. If animistic pronoun encounter, then it will refer to animistic referent. If non animistic occurs then it will refer to non animistic referent otherwise it will resolve pronoun with the use of Lappin Leass concept (With Recency factor). This model uses the concept of Lappin Leass for Recency factor. It is a hybrid approach. It uses a model that calculates the weight of a candidate based on different factors and uses this weight to rank potential candidates. The candidate with highest weight is used to resolve anaphors. The external knowledge is provided with the help of Gazetteer method.

Both our computational models use Gazetteer method for providing knowledge of Animistic factor. The difference in both the models is the use of Recency factor with different concepts (Centering approach and Lappin Leass approach). Based on these approaches results of experiments vary differently.

\section{EXPERIMENT AND RESULT}

\subsection{Data Set 1}

This experiment uses the text from children story domain. We have taken short stories in English language and performed anaphora resolution over these POS tagged stories. It contains approx 10 to 30 sentences having 100 to 400 words. Story 1 "The Thirsty Crow" is taken form moralstories.org (http://www.moralstories.org/the-thirsty-crow) and story 2 is taken from talesofpanchatantra.com http://www.talesofpanchatantra.com/shortstories_brahmins_dream.php). The result shown by experiment on both the model is summarized in table 1 and 2 . 
International Journal on Information Theory (IJIT), Vol.3, No.2, April 2014

Table1. Result of experiment performed by model 1 on short stories

\begin{tabular}{|l|l|l|l|l|l|}
\hline Data Set & $\begin{array}{l}\text { Total } \\
\text { Sentences }\end{array}$ & $\begin{array}{l}\text { Total } \\
\text { Word }\end{array}$ & $\begin{array}{l}\text { Total } \\
\text { Anaphors }\end{array}$ & $\begin{array}{l}\text { Correctly } \\
\text { Resolved } \\
\text { Anaphor }\end{array}$ & Accuracy \\
\hline Story1 & 16 & 165 & 12 & 12 & $100 \%$ \\
\hline Story2 & 28 & 364 & 50 & 33 & $66 \%$ \\
\hline
\end{tabular}

The result of proposed system 1 shows that Recency and animistic factor contribute 83\% accuracy to overall system.

Table2. Result of experiment performed by model 2 on short stories

\begin{tabular}{|l|l|l|l|l|l|}
\hline Data Set & $\begin{array}{l}\text { Total } \\
\text { Sentences }\end{array}$ & $\begin{array}{l}\text { Total } \\
\text { Word }\end{array}$ & $\begin{array}{l}\text { Total } \\
\text { Anaphors }\end{array}$ & $\begin{array}{l}\text { Correctly } \\
\text { Resolved } \\
\text { Anaphor }\end{array}$ & Accuracy \\
\hline Story1 & 16 & 165 & 12 & 12 & $100 \%$ \\
\hline Story2 & 28 & 364 & 50 & 36 & $72 \%$ \\
\hline
\end{tabular}

The result of proposed system 2 shows that Recency and animistic factor contribute $86 \%$ accuracy to overall system.

\subsection{Data Set 2}

This experiment uses text from news article domain. We have taken news articles from popular sites of English news. News 1 is taken from

(http://www.daijiworld.com/news/news_disp.asp?n_id=221629) and News 2 is taken from (http://www.siasat.com/english/news/modi-communal-wont-support-him-pm-mamata). The result of the experiment based on different model is summarized in table 3 and 4 .

Table3. Result of experiment performed by model 1 on news articles

\begin{tabular}{|l|l|l|l|l|l|}
\hline Data Set & $\begin{array}{l}\text { Total } \\
\text { Sentences }\end{array}$ & $\begin{array}{l}\text { Total } \\
\text { Word }\end{array}$ & $\begin{array}{l}\text { Total } \\
\text { Anaphors }\end{array}$ & $\begin{array}{l}\text { Correctly } \\
\text { Resolved } \\
\text { Anaphor }\end{array}$ & Accuracy \\
\hline News1 & 31 & 372 & 29 & 15 & $52 \%$ \\
\hline News2 & 20 & 387 & 42 & 29 & $69 \%$ \\
\hline
\end{tabular}

The result of proposed system 1 shows that Recency and animistic factor contribute $61 \%$ accuracy to overall system. 
International Journal on Information Theory (IJIT), Vol.3, No.2, April 2014

Table4. Result of experiment performed by model 2 on news articles

\begin{tabular}{|l|c|c|c|c|c|}
\hline Data Set & $\begin{array}{c}\text { Total } \\
\text { Sentences }\end{array}$ & $\begin{array}{c}\text { Total } \\
\text { Word }\end{array}$ & $\begin{array}{c}\text { Total } \\
\text { Anaphors }\end{array}$ & $\begin{array}{c}\text { Correctly } \\
\text { Resolved } \\
\text { Anaphor }\end{array}$ & Accuracy \\
\hline News1 & 31 & 372 & 29 & 21 & $73 \%$ \\
\hline News2 & 20 & 387 & 42 & 26 & $62 \%$ \\
\hline
\end{tabular}

The result of proposed system 2 shows that Recency and animistic factor contribute $68 \%$ accuracy to overall system.

\subsection{Data Set 3}

This experiment uses biography content from Wikipedia. We have taken biography of famous leaders of India from wikipedia.com, and then accuracy is calculated. The wiki 1 denotes the biography of Manmohan Singh taken from (http://en.wikipedia.org/wiki/Manmohan_Singh) and wiki 2 denotes the biography of Atal Bihari Vajpayee taken from (http://en.wikipedia.org/wiki/Atal_Bihari_Vajpayee). The result of experiment performed on both the system is summarized in table 5 and 6 .

Table5. Result of experiment performed by model 1 on Wikipedia content

\begin{tabular}{|c|c|c|c|c|c|}
\hline Data Set & $\begin{array}{c}\text { Total } \\
\text { Sentences }\end{array}$ & $\begin{array}{c}\text { Total } \\
\text { Word }\end{array}$ & $\begin{array}{c}\text { Total } \\
\text { Anaphors }\end{array}$ & $\begin{array}{c}\text { Correctly } \\
\text { Resolved } \\
\text { Anaphor }\end{array}$ & Accuracy \\
\hline Wiki1 & 18 & 368 & 9 & 7 & $78 \%$ \\
\hline Wiki2 & 10 & 280 & 13 & 11 & $85 \%$ \\
\hline
\end{tabular}

The result of proposed system 1 shows that Recency and animistic factor contribute $82 \%$ accuracy to overall system.

Table6. Result of experiment performed by model 2 on Wikipedia content

\begin{tabular}{|c|c|c|c|c|c|}
\hline Data Set & $\begin{array}{c}\text { Total } \\
\text { Sentences }\end{array}$ & $\begin{array}{c}\text { Total } \\
\text { Word }\end{array}$ & $\begin{array}{c}\text { Total } \\
\text { Anaphors }\end{array}$ & $\begin{array}{c}\text { Correctly } \\
\text { Resolved } \\
\text { Anaphor }\end{array}$ & Accuracy \\
\hline Wiki1 & 18 & 368 & 9 & 7 & $78 \%$ \\
\hline Wiki2 & 10 & 280 & 13 & 9 & $70 \%$ \\
\hline
\end{tabular}

The result of proposed system 2 shows that Recency and animistic factor contribute 74\% accuracy to overall system.

The correctness of the accuracy obtained by the experiment is measured by the language expert. From the above results it is concluded that the accuracy of the computational model depends on the genre of input data. For some input data, model 1 shows high accuracy whereas for other input data ,model 2 shows high accuracy. Hence the accuracy of the system depends on the type 
of input text. These features affect the overall accuracy. In order to increase accuracy more factors such as gender agreement, number agreement can be added to the system.

\section{Conclusion}

This paper presents the experimental results of two computational models for anaphora resolution. There are certain factors on which anaphora resolution depends, among which Recency and Animistic knowledge are adapted for our computational model. We have taken Recency as a constraint source which forms the base line of our experiment. The recognition of animacy entities is helpful to the promotion of accuracy. In this paper we have shown how anaphora resolution is done by performing experiment on different data sets. The experiment is performed to determine the contribution of Recency and animistic knowledge as a constraint sources to pronoun resolution on different styles of written text. The experiment conducted by Computational model 1 and 2 gives approx 70 to 80 percentage accuracy respectively. The accuracy of the model depends on the type of input context and the accuracy deviates according to the input data.

However, apart from Recency and animistic knowledge other sources like number agreement, gender agreement also play significant role in anaphora resolution. These factors affect the accuracy of both the systems. In the future we will try to incorporate these sources to further increase the accuracy of the overall system.

\section{References}

[1] Ruslan Mitkov, Richard Evans, (2007) “Anaphora Resolution: To What Extent Does It Help NLP Applications?” DAARC, LNAI 4410, pp. 179-190.

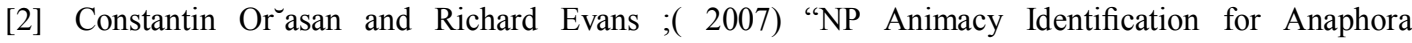
Resolution", Journal of Artificial Intelligence Research 29, 79-103.

[3] Razvan Bunescu,( 2003) “Associative anaphora resolution: A web-based approach”, In Proceedings of EACL 2003 - Workshop on The Computational Treatment of Anaphora, Budapest.

[4] Barlow, M., (1998), "Feature Mismatches and Anaphora Resolution", In Proceedings of DAARC2, University of Lancaster.

[5] Brent, (1993). "From grammar to lexicon: unsupervised learning of lexical syntax". Computational Linguistics, 19(3):243-262.

[6] Strube \& Hahn "A system for anaphora resolution for German based on extension of Centering theory".

[7] Thiago Thomes, "Lappin and Leass algorithm for pronoun resolution in Portuguese", Institute of State University of Campinas, Campinas, SP, Brazil, EPIA'05 Proceedings of the 12th Portuguese conference on Progress in Artificial Intelligence Pages 680-692.

[8] Aravind K Joshi, Rashmi Prasad, Eleni Miltsakaki "Anaphora Resolution: A Centering Approach".

[9] Dev Bahadur Poudel and Bivod Aale Magar "Anaphoric Resolution in Nepali”, Nepal Engineering College.

[10] Manuel Palomar, Lidia Moreno “Algorithm for Anaphora Resolution in Spanish Texts”, University of Alicante, Valencia University of Technology.

[11] McCord, Michael, (1990)"Slot grammar: A system for simpler construction of practical natural language grammars." In Natural Language and Logic: International Scientific Symposium, edited by R. Studer, 118-145. Lecture Notes in Computer.

[12] L. Sobha and B.N. Patnaik, "Vasisth: An anaphora resolution system for Malayalam and Hindi", Symposium on Translation Support Systems, 2002.

[13] K. Dutta, N. Prakash and S. Kaushik, "Resolving Pronominal Anaphora in Hindi using Hobbs algorithm," Web Journal of Formal Computation and Cognitive Linguistics, Issue 10, 2008.

[14] Anaphora Resolution in Tamil using Universal Networking Language "12/2011; In proceeding of: Indian International Conference on Artificial Intelligence (IICAI-2011), At Tumkur, Karnataka, India. 